medical needs of patients and families. There is urgent need to refine current $\mathrm{TH}$ protocols and encourage clinical trials of additional neuroprotective treatments.

\section{PILOT CASE SERIES ON THE USE OF MAGNESIUM SULPHATE AS NEUROPROTECTION IN HIE BABIES IN THE COOLING ERA}

doi:10.1136/archdischild-2012-302724.1111

WB Poon, SKY Ho. Neonatal and Developmental Medicine, Singapore General Hospital, Singapore, Singapore

Background and Aims A recent RCT suggested improved neurological outcome at discharge for moderate to severe perinatal asphyxia babies given iv magnesium sulphate. However, this trial was performed in babies who were not cooled.

Methods We present a pilot case series of 3 patients with moderate to severe HIE who satisfied the criteria for cooling and received both cooling and iv magnesium sulphate loading of $200 \mathrm{mg} / \mathrm{kg}$. Serum Magnesium levels were monitored at 0, 12, 24, 48, 72 hours of cooling.

The babies were reviewed for adverse effects of magnesium sulphate in terms of hypotension, arrhythmia, feed intolerance, respiratory depression and hypocalcemia.

Results One patient received systemic cooling and two other patients received selective head cooling. In addition to iv magnesium sulphate loading, decision was made to institute continuous infusion of iv magnesium sulphate in one of these patients for 4 days at $20-40 \mathrm{mg} / \mathrm{kg} / \mathrm{h}$ for PPHN. All babies achieved serum magnesium levels of $>1.2 \mathrm{mmol} / \mathrm{l}$ within $24 \mathrm{~h}$ of the loading dose, which was similar to the level aimed for in the previous RCT.

Magnesium sulphate was well tolerated with only mild hypotension requiring one day of dopamine $(\max 5 \mathrm{mcg} / \mathrm{kg} / \mathrm{min})$ in one patient. No babies had respiratory depression, arrhythmia, feed intolerance or hypocalcemia. Neurodevelopmental outcome to date is also presented.

Conclusions Magnesium sulphate is well tolerated in babies with moderate to severe HIE in the cooling era. A large RCT is required to assess its efficacy, long term impact and further look into adverse effects.

\section{2 \\ A TERTIARY NEONATAL UNIT'S EXPERIENCES IN ESTABLISHING AN IN-HOUSE COOLING SERVICE}

doi:10.1136/archdischild-2012-302724.1112

${ }^{1} \mathrm{R}$ Broughton, ${ }^{2} \mathrm{~V}$ Shaw, ${ }^{2} \mathrm{M}$ Borooah, ${ }^{3} \mathrm{SV}$ Rasiah. ${ }^{1}$ University of Birmingham; ${ }^{2}$ Neonatal Intensive Care Unit, Birmingham Women's Hospital NHS Foundation Trust; ${ }^{3}$ Neonatal Intensive Care Unit, Birmingham Women's Hospital NHS Foundation Trust, Birmingham, UK

Background Hypoxic Ischaemic Encepalopathy (HIE) affects 1-2 per 1,000 live births in UK. The TOBY study showed that therapeutic hypothermia $(\mathrm{TH})$ is beneficial for babies with moderate HIE. In view of this we established an in-house cooling service.

Aim To review our experiences in establishing an in-house cooling service.

Methods The Badger electronic database was used to identify babies who received TH in the last 2 years $(01 / 01 / 10$ to 31/12/11). The management and outcomes were analysed.

Results In the last two year, 27 babies' commenced in-house TH. 3 babies were transferred out to another unit for TH for bed capacity reasons. A further 3 babies died before discharge home. 19 babies received the full 72 hours of cooling in our centre. We did not encounter any major complications with the servo-controlled cooling mattress. Only $50 \%$ of babies had their MRI in the defined time period as per the TOBY guidelines. All babies are being followed up by a dedicated consultant Neonatologist and neurodevelopmental physiotherapist to assess their neurodevelopment up to the age of 2 years.

Conclusion We have safely established an in-house cooling service by following the TOBY guidelines. The servo-controlled cooling mattress provides a safe cooling process with a rectal probe. Identifying these babies early and the interpretation of CFAM was an important aspect of training. Our main challenge was to get an MRI post cooling in a timely fashion. This has been resolved with an agreed dedicated slot for these babies at Birmingham Children's Hospital.

\section{3D DIGITAL CAPTURE OF HEAD CIRCUMFERENCE AND VOLUME IN NEONATES - A METHOD EVALUATION}

doi:10.1136/archdischild-2012-302724.1113

S Ifflaender, M Rüdiger, A Koch, W Burkhardt. Department of Neonatology and Paediatric Intensive Care, University Hospital Carl Gustav Carus, Dresden, Germany

Background Manual measurement of head circumference (HC) is used to quantify head growth in preterm infants. Laser shape digitizers offer semi-automatic HC measuring and additional information on head volume (HV). Reliability and accuracy in obtaining $\mathrm{HC}$ and $\mathrm{HV}$ in neonates has not been investigated yet.

\section{Aims}

1. To determine intraobserver and interobserver variability of $\mathrm{HC}$ and $\mathrm{HV}$ measurements in neonates with a $3 \mathrm{D}$ digital capture system.

2. To compare the method with manual HC measurements

Methods Standard weekly HC measurements on a neonatal unit were conducted manually and digitally with STARScanner laser shape digitizer (Vorum Research Corp., Vancouver, BC) over 12 months. Method comparison was performed using Passing-Bablok-Regression (PBR), Cusum test and Bland-Altman (BA) analyses. Multiple scan examinations by different trained observers were performed to obtain intraobserver/interobserver data.

\section{Results}

1. Intraobserver coefficient of variation was low for $\mathrm{HC}$ (0.1$0.9 \%)$ and $\mathrm{HV}(0.54-1.1 \%)$. BA (mean percentage of difference $\left.M_{d^{\prime}} 95 \% \mathrm{CI}\right)$ of interobserver data showed interchangeability for HC $\left(M_{d}-0.005 ; C I-0.39-0.39\right)$ and $\mathrm{HV}\left(M_{d} 1.51 ; C I\right.$ $-1.17-4.1)$.

2. Method comparison data was acquired from 446 measurements in 258 infants (HC $318 \pm 19.5 \mathrm{~mm}$ ). Overall agreement was good $\left(M_{d}-0.82 ; C I-4.89-3.24\right)$, PBR showed no significant systematic or proportional differences $(a=1.03, \mathrm{CI}$ 0.99-1.06; $\beta=-7.06 C I-17.7-3.01)$. There was no significant deviation from linearity $(p=0.62)$

Conclusions Infant head shape capturing with the examined device is reliable, accurate and save. It offers additional information on HV. Possible benefits of HV in quantifying head growth in preterm infants need to be further investigated.

\section{RESUSCITATION WITH HYDROGEN GAS MIXED INTO SYNTHETIC AIR REDUCES APOPTOSIS IN NEONATAL HYPOXIA-ISCHEMIA PIGLET MODEL}

doi:10.1136/archdischild-2012-302724.1114

R Solberg, L Pankratov, OD Saugstad. Department of Paediatric Research, Os/o University Hospital - Rikshospitalet, Oslo, Norway

Background and Aims Asphyxia and subsequent reoxygenation cause a burst of oxygen free radicals. A gas mixture of ambient air and hydrogen may provide early neuroprotection.

Hydrogen may act as a therapeutic antioxidant by selectively reducing cytotoxic oxygen radicals and thereby contribute to less apoptosis. 
Methods Newborn piglets underwent hypoxia following a standardized model. They were randomly assigned for $30 \mathrm{~min}$ resuscitation with air $\left(21 \% \mathrm{O}_{2}\right)(\mathrm{n}=12)$ or $2.1 \%$ Hydrogen gas mixed into synthetic air, $\mathrm{H}_{2},(\mathrm{n}=14)$ and then observed for 9 hours. One control group $(n=6)$ went through the same procedures and observation time (anesthesia, surgery, ventilation and sample collection). The left hemisphere was used for histopathology. Tissue from prefrontal cortex and liver were snap frozen in liquid nitrogen and stored by $-70^{\circ} \mathrm{C}$ until analysis. The tissue samples were homogenized and the protein extracted. A Quantikine KM 300 immunoassay was used to measure activated caspase-3. protein. Gene expression for Casp-3, BDNF, MMP-2, MMP-9 and VEGFR2 was measured in tissue from prefrontal cortex and liver.

Results The use of $2.1 \%$ hydrogen gas mixed into synthetic air decreased activated caspase- 3 vs. air. In liver tissue piglets resuscitated with air: $12.6 \mathrm{pg} / \mathrm{mg}$ protein SD (9.1) vs. $\mathrm{H}_{2}: 5.3$ (4.9), $\mathrm{p}=0.031$ whereas in cortex piglets resuscitated with air $26.3 \mathrm{pg} / \mathrm{mg}$ protein (14.9) vs. $\mathrm{H}_{2} 15.4$ (13.0), $\mathrm{p}=0.05$.

There were no significant changes in gene expression in liver and cortex. Histopathology showed a tendency to less brain damage in the hydrogen group.

Conclusions Hydrogen gas used for newborn resuscitation may reduce apoptosis.

\section{THE NEUROPROTECTIVE EFFECTS OF VALPROIC ACID, AN HISTONE DEACETYLASE INHIBITOR IN A NEONATAL HYPOXIC-ISCHEMIC RAT MODEL}

doi:10.1136/archdischild-2012-302724.1115

'M Cetinkaya, 'T Alkan, 'M Cansev, 'Z Minbay, 'S Serter, 'E Orenlili, ${ }^{2} \mathrm{~N}$ Koksal. 'Uludağ University Medical Faculty, Bursa; 2 Uludağ University Medical Faculty, Ursa, Turkey

Introduction Neurodegenerative diseases were associated with a decrease in histone acetylase transferase (HAT) activity, resulting in relative over-deacetylation. Histone deacetylase (HDAC) inhibitors were suggested as potentially neuroprotective agents. The aim of this sudy was to evaluate the neuroprotective effects of valproic acid (VPA), an histone deacetylase inhibitor, in beonatal hypoxic ischemic rat model.

Methods After being anesthetized, 7-day-old pups underwent ischemia followed by exposure to hypoxia. The pups were divided into 3 groups: sham group, vehicle group (saline group) and VPA group. VPA was administered intraperitoneally for three times; the first just after hypoxia-ischemia, the second and the third doses 24 and 48 hours after the first dose, respectively. After sacrification; brain infarct volume, apoptosis, HDAC activity, acetylated $\mathrm{H} 4$ protein and caspase 3 expression, and proinflammatory cytokine concentrations were evaluated in brain tissue of rat pups. Results Percent infarcted brain volume and number of TUNEL positive cells per unit area in hippocampus and cortex CA1 were markedly reduced with VPA treatment. HDAC activity was found to be significantly reduced in VPA group, whereas acetylated H4 protein expression was significantly increased with VPA treatment. The caspase- 3 activity in VPA group was significantly lower than the control group. The roinflammatory cytokine levels also significantly decreased with VPA treatment.

Conclusion This is the first study that showed the neuroprotective effects of VPA treatment as an HDAC inhibitor by reducing percent infarcted brain volume, histone deacetylase activity, inflammation and apopotosis while increasing acetylated $\mathrm{H} 4$ protein levels in a neonatal hypoxic-ischemic rat model.

\section{6 \\ HYPERGLYCAEMIA AND INSULIN-INDUCED ALTERATIONS IN THE RETINA OF RAT PUPS}

doi:10.1136/archdischild-2012-302724.1116

'J Gyarmati, ${ }^{2} \mathrm{~K}$ Szabadfi, ${ }^{2} \mathrm{R}$ Gabriel, ${ }^{3 P}$ Kiss, ${ }^{3} \mathrm{D}$ Reglodi, 'B Mammel, 'T Kvarik, 'T Ertl. 'Department of Neonatology, Obstetrics and Gynaecology; ${ }^{2}$ Department of
Experimental Zoology and Neurobiology; ${ }^{3}$ Department of Anatomy, University of Pécs, Pécs, Hungary

Background and Aims Rat pups are applicable to investigate specific role of the factors which are implicated in the pathogenesis of retinopathy of prematurity ( $\mathrm{ROP}$ ) including hyperglycaemia and insulin treatment.

Methods The aim of our study was to investigate specific effect of streptozotocin-induced hyperglycaemia, insulin-treatment and intravitreal injection of a potential retinoprotective agent, pituitary adenylate cyclase activating polypeptide (PACAP) on the rat pups retina. We made a comparative analysis between the following treatment-groups: controls (Stz-/Ins-), insulin-treated (Stz-/Ins+), hyperglycaemic (Stz+/Ins-), insulin-treated hyperglycaemic (Stz+) Inz+); all animals were treated with intravitreal PACAP or vehicle. Blood glucose levels were monitored. The retinas were processed on P21 for routine histology and immunohistochemistry for glial fibrillary acidic protein (GFAP), GLUT1 and tyrosine hydroxylase (TH). Results Standard histological methods revealed no major differences between the groups. Elevated expression of GFAP - as an aspecific marker of metabolic insults in the retina- was detected from the inner retina in the Stz-/Ins+ group, although hypoglycaemia didn't develop. Similar alteration of the GFAP staining was found in the hyperglycaemic (Stz+/Ins-) and insulin-treated hyperglycaemic (Stz+/Inz+) groups. Intravitreal PACAP resulted in suppression of the elevated GFAP expression in the Stz-/Ins+ group, but not in the Stz+/Ins-, and Stz+/Inz+ ones. None of the groups showed alteration in the anti-TH immunoreactivity (dopaminergic amacrine cells) or GLUT1 expression of pigment epithelial cells.

Conclusions In our model hyperglycaemia or insulin did not induce ROP; however, sign of metabolic insult was detected in the neural retina, which was partly prevented by intravitreal PACAP application.

\section{A PIG MODEL OF THE PRETERM NEONATE: ANTHROPOMETRIC AND PHYSIOLOGICAL CHARACTERISTICS}

doi:10.1136/archdischild-2012-302724.1117

'YA Eiby, 'LL Wright, 1,2ER Lumbers, 'PB Colditz, 'BE Lingwood. ' $U 0$ Centre for Clinical Research, The University of Queensland, Brisbane, OLD; ${ }^{2}$ Mothers and Babies Research Centre, Hunter Medical Research Institute, Newcastle, NSW, Australia

Background and Aims Large animal models are an essential research tool to investigate the physiology of the preterm infant, which remains poorly understood. We aim to describe the pig model of the preterm neonate in terms of growth, maturation and requirement for intensive care over a range of gestational ages and determine the effects of maternal glucocorticoid exposure and sex.

Methods Twenty-nine litters of piglets $(\mathrm{N}=305)$ were delivered by C-section at 91d, 94d, 97d, 100d, 104d and 113d (term 115d). Some litters received maternal betamethasone treatment $(0.19 \mathrm{mg} / \mathrm{kg}$ body wt; IM) at $48 \mathrm{~h}$ and $24 \mathrm{~h}$ prior to delivery. At $97 \mathrm{~d}$ piglets were resuscitated, surfactant administered, and piglets were ventilated, sedated and monitored for 6-8h post-birth using standard NICU techniques. Results At 91d, piglets were half the weight of term animals, had fused eyelids, very thin skin, no hair, and survived a maximum of $3 \mathrm{~h}$ due to difficulties with ventilation. At $97 \mathrm{~d}$ piglets were able to be maintained for at least 6-8h but physiology was unstable for 1-2h. Piglets 100d and older breathed spontaneously. Only near term piglets were able to maintain body temperatures. Males were heavier than females at $113 \mathrm{~d}$ gestation $(\mathrm{p}=0.021)$. Exposure to maternal glucocorticoids resulted in larger females and influenced brain:body wt. Conclusions The piglet provides a useful model of preterm neonatal physiology as very preterm piglets can be survived under standard intensive care conditions. The large litters allow for parallel experiments or the use of littermates as controls. 